\title{
BMJ Open Development of a clinical prediction model to inform clinical decision making for classification of patients with sciatica, based on their clinical characteristics, in the Greek health system: protocol for a prospective predictive exploratory study
}

\author{
Nikolaos Kontakiotis (10 , ${ }^{1}$ Alison B Rushton (1) , ${ }^{2}$ Evdokia Billis, ${ }^{3}$ \\ George Papathanasiou, ${ }^{4}$ George Gioftsos (D) ${ }^{1}$
}

To cite: Kontakiotis N Rushton $\mathrm{AB}$, Billis $\mathrm{E}$, et al. Development of a clinical prediction model to inform clinical decision making for classification of patients with sciatica, based on their clinical characteristics, in the Greek health system: protocol for a prospective predictive exploratory study. BMJ Open 2022;12:e052119. doi:10.1136/ bmjopen-2021-052119

- Prepublication history for this paper is available online. To view these files, please visit the journal online (http://dx.doi. org/10.1136/bmjopen-2021052119).

Received 07 April 2021 Accepted 09 January 2022

Check for updates

(c) Author(s) (or their employer(s)) 2022. Re-use permitted under CC BY-NC. No commercial re-use. See rights and permissions. Published by BMJ.

For numbered affiliations see end of article.

Correspondence to Nikolaos Kontakiotis; nkontakiotis@uniwa.gr

\section{ABSTRACT}

Introduction Sciatica is one of the most common reasons for seeking healthcare for musculoskeletal pain. Sciatica is primarily considered as neuropathic in nature when neural tissue in the low back is compromised, but sometimes other non-neural structures may be involved. Appropriate assessment and management are important for patients with sciatica. Therapists use several outcome measures to assess patients to inform selection of the most suitable treatment. There is limited evidence for the best treatment of sciatica, and this is likely contributed to by having no reliable algorithm to categorise patients based on their clinical characteristics to inform physiotherapy treatment. The purpose of this study is to develop a clinical prediction model to categorise patients with sciatica, in terms of early clinical outcome, based on their initial clinical characteristics.

Methods and analysis A prospective observational multicentre design will recruit consecutive patients $(n=467)$ with sciatica referred for physiotherapy. Each patient will be evaluated to determine whether or not they will be accepted into the study by answering some questions that will confirm the study's eligibility criteria. Patients' basic characteristics, patient-reported outcome measures and performance-based measures will be collected at baseline from multiple sites in the Greek territory using this same protocol, prior to commencement of treatment. The main researcher of this study will be responsible for data collection in all sites. On completion of the standard referred physiotherapy treatment after 3 weeks' time, participants will be asked by telephone to evaluate their outcome using the Global Perceived Effect Scale. For the descriptive statistical analysis, the continuous variables will be expressed in the form of 'mean' and 'SD'. In order to assess the prognostic value of each predictor, in terms of the level of improvement or worsening of the symptoms, multiple variable regression analysis will be used.

\section{Strengths and limitations of this study}

This will be the first research study in Greece to categorise patients with sciatica, in terms of clinical outcome, based on their clinical characteristics. It will generate a fast and reliable algorithm for every day clinical practice for healthcare professionals that can predict the early outcome of the patients after physiotherapy treatment based on their clinical characteristics.

- Candidate predictive factors selected from a systematic review, other published studies and experts' opinions.

- This prospective predictive study will be conducted according to the TRIPOD checklist (Prediction Model Development), PROGRESS framework and PROBAST.

- For reliability reasons, the main researcher will conduct all data collection to all participants to ensure standardised procedures of data collection.

- The outcome will be dichotomised and that maybe will lead to loss of information and risk of misclassification.

- Due to COVID-19 restrictions during the planned period of the study, this may create difficulties for gathering a larger sample.

Ethics and dissemination This study is approved from the Ethics and Deontology Committee of the University of West Attica, Athens, Greece, protocol number: 38313-09/06/2020, 10226-10/02/2021. The study's findings will be published in a peer-reviewed journal and disseminated at national and international conferences and through social media. PROSPERO registration number CRD42020168467.

\section{INTRODUCTION}

In primary care, a large proportion of patients with low back pain (LBP), approximately $60 \%$, 
also report low back-related leg pain (LBLP). ${ }^{1}$ This pain is commonly referred to as sciatica and is often described as pain radiating to the buttocks, thighs and below the knee, foot and/or toes. LBLP is generally clinically diagnosed as sciatica (lumbar radicular) or referred pain (involving non-neural structures); sciatica is considered neuropathic in nature whereas referred pain is considered nociceptive. ${ }^{2}$ It may be accompanied by physical findings of nerve root entrapment, such as decreased sensitivity, alteration in reflex and/or muscle weakness in entrapped nerve root distribution. ${ }^{3}$ However, there is evidence to suggest the coexistence of both pain mechanisms in LBLP, ${ }^{4}$ and evidence for sciatica presenting without neuropathic pain and referred pain presenting with neuropathic pain. ${ }^{5}$ The prevalence of neuropathic pain reported in a recent study by Harrisson et $a l^{2}$ estimates of neuropathic pain in LBLP ranged from $19 \%$ to $80 \%$, although these findings must be observed with caution as the review itself is at moderate risk of bias. LBLP compared with LBP alone is associated with increased disability, pain and poorer quality of life. ${ }^{6}$ Sciatica is a relatively common and often a persistent "nuisance" ${ }^{7}$ that leads to the use of health services, ${ }^{8}$ prolonged sick leave ${ }^{9}$ and has considerable economic consequence in terms of healthcare resources and lost productivity. ${ }^{10}$ Although prognosis is good for most patients, a significant proportion (up to $30 \%$ ) still have pain for a year or more. ${ }^{11}$

The aetiology of sciatica varies as described above, as well as lumbar radiculopathy and referred pain. It may be due to severe vertebral pathology as well as other non-vertebral diseases. ${ }^{12}$ In a large proportion of cases, it is caused by an intervertebral disc herniation resulting in nerve root irritation, ${ }^{3}$ but yet in these cases the management is not straight forward. Sciatica is, therefore, a broad term encompassing multiple diagnoses that require different management. ${ }^{3}$ Evaluation of aetiology often involves the use of expensive imaging methods (MRI) which can lead to wrong conclusions regarding the causes of pain. ${ }^{12}$ The diagnosis and management of sciatica varies considerably within and between countries, ${ }^{3}$ which may reflect treatment availability, clinician preference and socioeconomic variables rather than evidence-based practice. Previous systematic reviews (including meta-analyses) have evaluated the effectiveness of various individual treatment approaches for sciatica, including conservative treatments, ${ }^{11}{ }^{13-15}$ epidural steroid injections ${ }^{11} 141617$ and surgical procedures. ${ }^{18}$ However, numerous treatments have not been directly compared with other interventions and the results have not been statistically or clinically significant. In order to compare interventions, it would be helpful if study populations had the same clinical characteristics. Populations in the studies are commonly heterogeneous owing to the multiple diagnoses and aetiopathological parameters within sciatica. It is thus easily understood that research interest is increasingly focused on exploring more effective evaluation tools to categorise these patients based on their clinical characteristics with the aim of prediction the outcome of the patients after physiotherapy treatment.
Although there is worldwide research interest in possible classification prediction models for these patients, there is an urgent need to develop a clinical and research application algorithm for Greek society for clinical and research use. In the international physiotherapy community, some evaluation tools for sciatica have been proposed with sometimes poor, controversial and/or conflicting results. ${ }^{12}$ Although the sample of these research was sufficient the results cannot be generalised to other health systems such as the Greek. There are psychosocial differences in patients of different nationalities in terms of pain and its treatment. ${ }^{19}$ and international systems may not be appropriate. Culture across countries also varies. Culture according to Helman ${ }^{20}$ is a set of guidelines that individuals inherit as members of a particular society that tells them how to see the world, how to experience it emotionally and how to behave in relation to other people. There is evidence that there are cultural differences in medical, physical and psychosocial findings among patients with pain in the low lumbar region. ${ }^{1921}$ and research based on intracultural comparisons could provide valuable information on the development of features to be included in a classification system. ${ }^{22}$ In Greece, no study has yet investigated the evaluation and categorisation of patients with sciatica in terms of early clinical outcome, based on their initial clinical characteristics.

\section{Objective}

To develop a clinical prediction model to categorise patients with sciatica in terms of early clinical outcome, based on their initial clinical characteristics.

\section{METHODS AND ANALYSIS \\ Source of data}

This research is a prospective predictive exploratory observational multicentre study for clinical prediction model development within the Greek health system. Patients with sciatica referred for physiotherapy will be recruited. The study will be started on June 2021 with anticipated completed date on September 2022. Selfreported and physical examination potential predictive data items will be collected at baseline prior to treatment, which will commence immediately following recruitment. Following collection of potential predictive data items, patients will undergo physiotherapy treatment. At the end of their standard physiotherapy treatment, at 3 weeks postbaseline, outcome will be evaluated. Outcome will be assessed using Global Perceived Effect Scale (GPES). This protocol is written in line with the transparent reporting of a multivariable prediction model for individual prognosis or diagnosis (TRIPOD) statement of items that should be included in reports of prediction model development and validation. ${ }^{23}$ Prognosis research strategy (PROGRESS) framework ${ }^{24}$ and prediction model risk of bias assessment tool (PROBAST) ${ }^{25}$ were used for the plan analysis of this study. 


\section{Participants and eligibility}

Consecutive eligible patients will be recruited in order not to introduce bias. Participants will be patients with diagnosed sciatica, referred from spinal surgeons for standard physiotherapy provided by their public insurance and will contribute to our understanding of the findings from the systematic review informing this application. Patients may have with them X-ray and/or MRI results of investigations of their back. Participants will be recruited from private physiotherapy clinics in Greece. Physiotherapists, owners of these clinics, will be invited to participate as a clinical site in the research. All consecutive eligible patients will be approached for recruitment into the study by the clinic owner who will be responsible to book the appointment for the assessment. The special musculoskeletal physiotherapist that is the main researcher of this study will be informed for the appointment and will go to each clinic for the data collection. Based on feasibility data, it is estimated that 30 eligible participants will be available for recruitment in a 1-month period across sites. Initially, each patient will be evaluated to determine whether or not they will be accepted into the study by answering some simple questions that will confirm the study's eligibility criteria. All patients will be asked to provide written informed consent for participating to the study, they will consent for publication of the results and they will be informed that they allowed to withdraw from the study at any time they wish without impact on their management. A lay summary of the study report will be written for patients and disseminated through social media, conferences and publication to raise awareness of the study and future research plans.

Inclusion criteria:

- Patients with sciatica diagnosed by a spine specialist in a private or public hospital/clinic and referred to standard physiotherapy using their public health insurance.

- Pain in the buttock region that refers into the lower extremity in any distribution.

- Aged 18-75 years old.

- Ability to read and communicate in Greek.

Exclusion criteria:

- Suspected serious spinal pathology or clinical redflags such as cauda equina syndrome, suspicion of spinal tumours, infection, fractures and inflammatory spondyloarthropathy.

- Previous lumbar spine surgery.

- Previous lower extremity surgery.

- Currently receiving ongoing care from or have been in consultation with a secondary care doctor or physiotherapist for the same problem in the last 3 months.

- Serious comorbidity preventing them from attending the research clinic and/or undergoing assessment and interventions.

- Severe enduring mental health condition.

- Pregnancy.

- Current participation in any other research study because of symptoms of back and leg pain or sciatica.
- Scoliosis of the spine $>10$ degrees (Cobb's angle), as measured on an X-ray.

\section{Candidate predictors}

All participants will be assessed at baseline by the main researcher to ensure reliability of the procedure. Patients' basic characteristics including age, gender, weight, height will be recorded. In addition, a range of (1) patient-reported outcome measures (PROMs) and (2) performance-based measures (PBOs) to assess sciatica will be collected. PROMs used in this study have been translated and cultural adapted into Greek and have been investigated for their reliability and validity. ${ }^{26-33}$ The summary of the candidate predictor factors is shown in table 1 . These factors have potential predictive ability identified from a recent systematic review (manuscript under review) investigating the diagnostic value of PROMs and PBOs used to assess patients presenting with sciatica and previous predictive studies. ${ }^{34}$ Finally, expert's opinion informed the final list of candidate predictors.

\section{Outcome}

GPESs are broadly used in clinical practice to assess patient outcome following intervention. They are used for several musculoskeletal conditions and in many languages. In this study the GPES-7 GR will be used to assess outcome at 3 weeks postbaseline and after the completion of their standard physiotherapy. The 3-week follow-up was chosen to be immediately after the completion of the standard physiotherapy treatment provided by public health insurance in Greece. The GPES-7 GR was translated and assessed for reliability in a previous study (manuscript under review) in patients with sciatica in Greece and showed excellent test-retest reliability. In the analysis of the test-retest reliability, the GPES-7 showed excellent agreement with $\mathrm{k}=0.919$ (95\% CI 83.3 to 92). The following question of the GPES-7 GR will be asked: To what extent are your complaints changed when compared with the situation just before you started treatment? The possible answers, to the question, are 7: '(1) completely recovered, (2) much improved, (3) slightly improved, (4) no change, (5) slightly worsened, (6) much worsened and (7) worse than ever.' Results will be dichotomised such that 'completely recovered' and 'much improved' will be categorised as 'improved'. 35

\section{Sample size}

In predictive modelling a larger sample size enables lower bias and variance, and permits the prospective prediction of new observations. ${ }^{36}$ The minimum required sample was calculated, equal to 420 patients because for a predictive study there is a minimum framework for 10 participants per predictor. In order to cover a possible loss of $10 \%$ of patients, the inclusion of a total sample of participants was set to $\mathrm{n}=467 .{ }^{37}$

\section{Methodological quality in the prediction model}

Limited research has identified criteria for quality in a prediction model, but authors have identified potential 
Table 1 Summary of candidate predictors to be collected at baseline

\begin{tabular}{ll}
\hline Candidate predictor & Measure/data item and how recorded \\
\hline \multicolumn{2}{l}{ General patient characteristics } \\
$\mathrm{Age}^{34}$ & In years \\
$\mathrm{Gender}^{34}$ & $\begin{array}{l}\text { Female/male/other } \\
\mathrm{BMI}^{34}\end{array}$ \\
$\begin{array}{l}\text { Calculated from height and weight } \\
\text { measurements }\end{array}$ \\
$\begin{array}{l}\text { Pain duration } \\
\text { Pain below knee }\end{array}$ & $>3$ weeks $>6$ weeks $>3$ months \\
\hline $\begin{array}{l}\text { Pain after coughing / } \\
\text { sneezing }\end{array}$ & Yes/no \\
MRI $^{34}$ & Yes/no \\
& $\begin{array}{l}\text { Clear/possible nerve root compression/ } \\
\text { disc prolapse/stenosis/other }\end{array}$
\end{tabular}

\begin{tabular}{ll}
\hline PROMs & Oswestry Disability Index ${ }^{26}$ \\
\hline Disability & Sciatica Bothersomeness Index ${ }^{27}$ \\
$\begin{array}{l}\text { Overall impact of sciatica } \\
\text { symptoms }\end{array}$ & Tampa scale of kinesiophobia ${ }^{28}$ \\
$\begin{array}{l}\text { Fear of movement or fear } \\
\text { of injury or re-injury during } \\
\text { movement }\end{array}$ &
\end{tabular}

\begin{tabular}{|c|c|}
\hline Anxiety and depression & $\begin{array}{l}\text { Depression Anxiety and Stress } \\
\text { Scale- } 21^{29}\end{array}$ \\
\hline Neuropathic pain & $\begin{array}{l}\text { Leeds Assessment of Neuropathic } \\
\text { Symptoms and Signs }{ }^{30}\end{array}$ \\
\hline $\begin{array}{l}\text { Central sensitisation/central } \\
\text { sensitivity syndromes }\end{array}$ & Central Sensitisation Inventory ${ }^{31}$ \\
\hline Pain intensity & Short-form McGill Pain Questionnaire 32 \\
\hline Quality of life & Short Form-12 ${ }^{33}$ \\
\hline \multicolumn{2}{|l|}{ PBOs } \\
\hline Straight Leg Raise & Sciatica symptoms \\
\hline Muscle strength & Neurological examination \\
\hline Reflexes & $\begin{array}{l}\text { Neurological examination (quadricep } \\
\text { tendon/Achilles' tendon) }\end{array}$ \\
\hline Heat pain threshold & $\begin{array}{l}\text { Evaluation of pain threshold using a } \\
\text { heat stimulus }\end{array}$ \\
\hline Cold pain threshold & $\begin{array}{l}\text { Evaluation of pain threshold using a } \\
\text { cold stimulus }\end{array}$ \\
\hline Vibration pain threshold & $\begin{array}{l}\text { Evaluation of pain threshold using a } \\
\text { tuning fork }\end{array}$ \\
\hline Pin prick response & $\begin{array}{l}\text { Evaluation of pinch sensation using a } \\
\text { pin prick }\end{array}$ \\
\hline Light touch sensation & $\begin{array}{l}\text { Evaluation of light touch sensation } \\
\text { using a cotton }\end{array}$ \\
\hline $\begin{array}{l}\text { Two-point discrimination } \\
\text { sensation }\end{array}$ & $\begin{array}{l}\text { Evaluation of two-point discrimination } \\
\text { sensation using a discrimination two- } \\
\text { point tool }\end{array}$ \\
\hline
\end{tabular}

BMI, body mass index; PBOs, performance-based measures; PROMs, patient-reported outcome measures.

quality issues to ensure methodological rigour. ${ }^{38}$ All issues have been addressed/planned for in this study (table 2).

\section{Data collection}

This study is coproduced by patients with sciatica, the specialist musculoskeletal physiotherapist which is the main researcher of the study and healthcare professionals over Greece that are participating as clinical sites.
Patients will be examined only by the specialist musculoskeletal physiotherapist that is the main researcher of this study. The researcher will collect and record all data. The baseline assessment will consist of PROMs, neurological examination and Straight Leg Raise (SLR) to ensure all candidate predictors are recorded. Self-reported questionnaires will be completed in the presence of the specialist musculoskeletal physiotherapist to avoid any queries. The specialist musculoskeletal physiotherapist will perform the neurological examination and quantitative sensory tests with the tool kit to draw conclusions about the neurological dysfunction of each patient. Muscle strength of the lower quadrant and reflexes of quadricep's tendon and Achilles' tendon will be evaluated. The tool kit will include a coin for the hot/ cold sensatio, ${ }^{39}$ a tuning fork for vibration, a pin prick for pinch sensation, a soft tissue for light touch and a discrimination point tool. The SLR will be considered as positive when the symptoms of the patient will be reproduced from the test. If the SLR is positive the specialist musculoskeletal physiotherapist will then measure the angle of hip flexion at which the symptoms occurred. The angle will be measured with a goniometer pro app (G-PRO) for IOS and android. ${ }^{40}$ All patients will undergo standard physiotherapy provided by their public insurance with 3 weeks duration. This physiotherapy consists of electrotherapy, massage therapy and exercises. Outcome will be assessed at 3 weeks postbaseline on completion of the standard physiotherapy by answering the following question of the GPES-7GR: To what extent are your complaints changed when compared with the situation just before you started treatment? The possible answers, to the question, are 7: - (1) completely recovered, (2) much improved, (3) slightly improved, (4) no change, (5) slightly worsened, (6) much worsened and (7) worse than ever.' The specialist musculoskeletal physiotherapist that is the main researcher of this study will contact by telephone each patient and will be recording their answer.

\section{Statistical methods and management of missing data}

Potentially eligible patients, numbers examined for eligibility, confirmed eligible, recruited into the study, completing follow-up and analysed will be reported in a flow diagram. The diagram will illustrate the flow of participants through the study, including the number of participants with and without the outcome, and capturing all time points. The correlation between candidate predictive factors will be calculated at baseline. Reasons for non-participation, exclusion, drop-outs and withdrawal will be reported at each stage. For the descriptive statistical analysis, the continuous candidate predictors will be expressed in the form of 'mean' and 'SD'. The characteristics of the participants (demographics, clinical features, predictors) will be reported, with clarity of the number of participants with missing data for either outcome or predictors. For each variable of interest, the number of participants with missing data will be reported. 
Table 2 Methodological decisions to improve quality in prediction models

\begin{tabular}{|c|c|}
\hline Criteria $^{17}$ & Methodological decisions to improve quality \\
\hline \multicolumn{2}{|l|}{ Study design } \\
\hline Inception cohort & $\begin{array}{l}\text { Clear description of population. } \\
\text { Clear description of the participants at baseline. }\end{array}$ \\
\hline Source population & $\begin{array}{l}\text { Clear description of population. } \\
\text { Clear description of sampling frame and recruitment } \\
\text { (method and timing). }\end{array}$ \\
\hline Inclusion and exclusion criteria & - Clarity of eligibility criteria. \\
\hline Prospective design & Clarity of study design \\
\hline \multicolumn{2}{|l|}{ Study attrition } \\
\hline No of drop-outs & $\begin{array}{l}\text { Adequate participation rate. } \\
\text { Clear description of attempts to collect information on } \\
\text { participants who dropped out. }\end{array}$ \\
\hline $\begin{array}{l}\text { Information provided on method of management of missing } \\
\text { data }\end{array}$ & Appropriate methods of imputation of missing data. \\
\hline
\end{tabular}

Predictive factors

All predictive factors described used to develop the model $\rightarrow$ Clear definition of predictive factors.

- An adequate proportion of participants has completed data for the predictive factor.

$\begin{array}{ll}\text { Standardised or valid measurements } & \text { The measurement of the predictive factor is reliable and } \\ & \text { valid. } \\ \text { Linearity assumption studied } & \text { The measurement of the predictive factor is the same for all } \\ \text { No dichotomisation of predictive variables } & \text { Linearity of data will be reported. } \\ \text { Data presentation all predictive factors } & \text { Continuous variables will be reported. } \\ \text { Outcome measures } & \text { Complete data will be presented. } \\ \text { Description of outcome measures } & \text { The outcome is clearly defined. } \\ \text { Standardised or valid measurements } & \text { The measurement of the outcome is reliable and valid. } \\ & \text { participants. } \\ \text { Data presentation of most important outcome measures } & \text { Complete data will be presented. }\end{array}$

\begin{tabular}{ll} 
Analysis & An appropriate strategy for model building is described. \\
Presentation of univariate crude estimates & An adequate statistical model described. \\
Sufficient numbers of subjects per variable & Adequate data will be presented. \\
\hline Selection method of variables explained & Sufficient data will be presented to enable assessment of \\
& the adequacy of the analytic strategy. \\
Presentation of multivariate estimates & All results will be reported. \\
\hline Clinical performance/validity & An appropriate strategy for model building is described. \\
Clinical performance & An adequate statistical model described. \\
Internal validation & Clinical performance of the model will be reported. \\
External validation & Internal validation will be reported
\end{tabular}

Statistical modelling for prediction has been planned a priori. To explore the influence of each predictive factor, in terms of the level of improvement or worsening of the symptoms, a logistic multivariable regression model will be fitted to the dichotomised outcome scores to calculate outcome. ORs for each candidate predictive factor will be reported, adjusted for other factors and account for clustering. Multivariable analysis will initially include all candidate predictive factors, and full results reported. If necessary, multiple imputation. ${ }^{41}$ will be used to deal 
with missing data. Reduced multivariable analyses will be considered if necessary (eg, removing one of two candidate predictive factors that are highly correlated at baseline), to examine the robustness of the conclusions. Selection of items for the model will include those factors which are statistically significantly $(\mathrm{p}<0.05)$ associated with poor outcome according to the full multivariable regression analysis using backward stepwise selection. ${ }^{23}$ and those deemed clinically important to retain (regardless of statistical significance) to improve face validity for clinicians. The regression model with included predictive factors will be fitted to the cohort data to obtain a final set of parameter estimates (ie, alpha and beta terms) which will be used to form the model. Cross-validation will be used for internal validation of the model. Cross-validation can be seen as an extension of the split-sample approach. In a 10 -fold cross-validation, the model is developed on $90 \%$ of the population and tested in the remaining $10 \%$. This is repeated 10 times, each time using another $10 \%$ of the population for testing so that all patients have been included in the test group once. Internal validation will provide estimates of the ability of the model to discriminate between patients with different outcomes as well as the agreement between the observed and predicted risks (calibration). External validation of the derived model is not a focus of this study, because this clinical prediction model targets to patients with sciatica treating with physiotherapy in the Greek heath system. External validation would be very useful if this model was targeted in other patient samples; other physiotherapy settings; wider range of therapists; other rehabilitations techniques and other populations. Also, a new bigger sample is needed for this analysis. Due to COVID-19 restrictions unfortunately, this sample cannot be gathered. A further research in the future with larger sample will address the external validity. All data will be analysed using IBM SPSS V.25 Statistics.

\section{Patients and public involvement}

Patients and the general public were not involved in the development of the research questions, the design, recruitment and implementation of this study. However, the study participants will be informed for the results of this study through a peer-reviewed journal, national and international conferences and through social media.

\section{ETHICAL CONSIDERATIONS}

All patients before the procedure will be informed by the specialist musculoskeletal physiotherapist about the purpose of this study by an information sheet and about the assessment that they will experience. After that and before the beginning of the assessment, they will have the chance to ask questions about the procedure, they will sign a consent form and they will be given with a separate form for any complaints that may have. Confidentiality due to sensitive personal data will be secured during the study, but also after publication of the results. Finally, they will consent for publication of the results and they will be informed that they allowed to withdraw from the study at any time they wish without any implication.

\section{Ethical approval}

The study is approved from the Ethics and Deontology Committee of the University of West Attica, Athens, Greece, protocol number: 38313-09/06/2020, 10226-10/02/2021.

\section{Privacy of information}

All data collected will be kept confidentially, and only the main researcher will have access to it. Raw data initially collected by patients will be stored in excel format on the main researcher's personal computer where only the one with security codes will have access. An excel-type backup file will be created on an external disk, accessible only by the main researcher with security codes. No other member of the research team will have access to data. The original raw files will then be destroyed.

\section{DISCUSSION}

Many treatments have been investigated to treat people with sciatica, but none have proven statistically or clinically significant. ${ }^{42}$ An explanation for this unsuccessful treatment may be related to the inability to differentiate the problem clinically, which in turn contributes to the lack of classification of these patients into subgroups according to their clinical characteristics. Individual remarkable efforts highlight the necessity of this issue and offer the first relevant information limited to a specific health system and population. ${ }^{34}$ The categorisation of Stynes $e t a l^{34}$ was limited to the English public health system and in particular to the primary healthcare setting. This study will be the first in Greece, and aims to categorise patients with sciatica in terms of early clinical outcome based on their initial clinical characteristics in the Greek health system. Data analysis and expert opinion from the field will be taken into consideration for potential classification of patients as low/high or low/medium/ high-risk probability to have a successful outcome with physiotherapy. As a limitation of this study is that the outcome will be dichotomised and that maybe will lead to loss of information and risk of misclassification. Data will be collected from multiple physiotherapy clinics across Greece. The main researcher of this study will be responsible for data collection in all sites and to gain reliability of all the PBOs maybe there is a bias in performing them. For that reason, this is a limitation of this study. Another limitation is that due to COVID-19 restrictions during the planned period of the study, this may create difficulties for gathering a larger sample. Despite these accepted limitations, this study will enable important insights into this important subject. Procedures are already in place to enable data collection through physiotherapy clinics.

\section{Clinical implication}

This study will enable predictions to be made regarding the outcome of the physiotherapy treatment of patients 
with sciatica based on their clinical characteristics. That will happen through classification of these patients based on their clinical characteristics and will enable initial decision making in primary care of the Greek health system. The predictive model that will be developed will be fully adapted to the Greek reality and will lead to the selection of the most useful predictors for improving the clinical effectiveness of daily specialised physiotherapeutic practice in patients with sciatica. Future implementation of the prediction model will enable patients' classification based on their clinical characteristics that may predict the outcome of the patients after physiotherapy treatment. The clinical application of the prediction model will be easy to use, fast and will provide a sufficient way of assessing this population. It will also promote communication between health professionals (especially doctors and physiotherapists) managing populations with sciatica.

\section{DISSEMINATION PLAN}

The study's findings will be published in a peer-reviewed journal and disseminated at national and international conferences and through social media.

\section{Author affiliations}

${ }^{1}$ Physiotherapy Department, Laboratory of Advanced Physiotherapy, Faculty of Health and Caring Sciences, University of West Attica, Athens, Greece

${ }^{2}$ School of Physical Therapy, Western University Faculty of Health Sciences, London, Ontario, Canada

${ }^{3}$ Physiotherapy Department, School of Health Rehabilitation Sciences, University of Patras, Patra, Greece

${ }^{4}$ Physiotherapy Department, Laboratory of Neuromuscular and Cardiovascular Study of Motion, Faculty of Health and Caring Sciences, University of West Attica, Athens, Greece

\section{Twitter Alison B Rushton @abrushton}

Contributors NK led the design of the study as a doctoral research supervised by GG. ABR drafted the manuscript and provided feedback on the manuscript. EB and GP will contribute to data interpretation, conclusions and dissemination. All authors approved the final version.

Funding The authors have not declared a specific grant for this research from any funding agency in the public, commercial or not-for-profit sectors.

Competing interests None declared.

Patient and public involvement Patients and/or the public were not involved in the design, or conduct, or reporting, or dissemination plans of this research.

Patient consent for publication Not applicable.

Provenance and peer review Not commissioned; externally peer reviewed.

Open access This is an open access article distributed in accordance with the Creative Commons Attribution Non Commercial (CC BY-NC 4.0) license, which permits others to distribute, remix, adapt, build upon this work non-commercially, and license their derivative works on different terms, provided the original work is properly cited, appropriate credit is given, any changes made indicated, and the use is non-commercial. See: http://creativecommons.org/licenses/by-nc/4.0/.

\section{ORCID iDs}

Nikolaos Kontakiotis http://orcid.org/0000-0001-9779-8570

Alison B Rushton http://orcid.org/0000-0001-8114-7669

George Gioftsos http://orcid.org/0000-0002-3368-3114
REFERENCES

1 Hill JC, Konstantinou K, Egbewale BE, et al. Clinical outcomes among low back pain consulters with referred leg pain in primary care. Spine 2011;36:2168-75.

2 Harrisson SA, Stynes S, Dunn KM, et al. Neuropathic pain in low Back-Related leg pain patients: what is the evidence of prevalence, characteristics, and prognosis in primary care? A systematic review of the literature. J Pain 2017;18:1295-312.

3 Koes BW, van Tulder MW, Peul WC. Diagnosis and treatment of sciatica. BMJ 2007;334:1313-7.

4 Freynhagen R, Rolke R, Baron R, et al. Pseudoradicular and radicular low-back pain--a disease continuum rather than different entities? Answers from quantitative sensory testing. Pain 2008;135:65-74.

5 Mahn F, Hüllemann P, Gockel U, et al. Sensory symptom profiles and co-morbidities in painful radiculopathy. PLoS One 2011;6:e18018.

6 Konstantinou K, Hider SL, Jordan JL, et al. The impact of low backrelated leg pain on outcomes as compared with low back pain alone: a systematic review of the literature. Clin J Pain 2013;29:644-54.

7 Konstantinou K, Dunn KM. Sciatica: review of epidemiological studies and prevalence estimates. Spine 2008;33:2464-72.

8 Molano SM, Burdorf A, Elders LA. Factors associated with medical care-seeking due to low-back pain in scaffolders. Am J Ind Med 2001;40:275-81.

9 Hagen KB, Thune O. Work incapacity from low back pain in the general population. Spine 1998;23:2091-5.

10 Samanta A, Beardsley J. Evidence based case report: sciatica: which intervention? BMJ 1999;319:302-3.

11 Vroomen PC, de Krom MC, Slofstra PD, et al. Conservative treatment of sciatica: a systematic review. J Spinal Disord 2000;13:463-9.

12 Stynes S, Konstantinou K, Dunn KM, et al. Reliability among clinicians diagnosing low back-related leg pain. Eur Spine $J$ 2016;25:2734-40.

13 Hagen KB, Hilde G, Jamtvedt G, et al. Bed rest for acute low-back pain and sciatica. Cochrane Database Syst Rev 2004:CD001254.

14 Luijsterburg PAJ, Verhagen AP, Ostelo RWJG, et al. Effectiveness of conservative treatments for the lumbosacral radicular syndrome: a systematic review. Eur Spine J 2007;16:881-99.

15 Clarke JA, van Tulder MW, Blomberg SEl. Traction for low-back pain with or without sciatica. Cochrane Database Syst Rev 2007;2.

16 Boswell MV, Hansen HC, Trescot AM, et al. Epidural steroids in the management of chronic spinal pain and radiculopathy. Pain Physician 2003;6:319-34.

17 Abdi S, Datta S, Trescot AM, et al. Epidural steroids in the management of chronic spinal pain: a systematic review. Pain Physician 2007;10:185-212.

18 Gibson JNA, Waddell G. Surgical interventions for lumbar disc prolapse. Spine 2007;32:1735-47.

19 Sanders SH, Brena SF, Spier CJ, et al. Chronic low back pain patients around the world: cross-cultural similarities and differences. Clin J Pain 1992;8:317-23.

20 Helman $\mathrm{CH}$. Culture health and illness. 5th edition. Butterworth London, 2007.

21 Carron H, DeGood DE, Tait R. A comparison of low back pain patients in the United States and New Zealand: psychosocial and economic factors affecting severity of disability. Pain 1985;21:77-89.

22 Billis EV, McCarthy CJ, Stathopoulos I, et al. The clinical and cultural factors in classifying low back pain patients within Greece: a qualitative exploration of Greek health professionals. J Eval Clin Pract 2007;13:337-45.

23 Moons KGM, Altman DG, Reitsma JB, et al. Transparent reporting of a multivariable prediction model for individual prognosis or diagnosis (TRIPOD): explanation and elaboration. Ann Intern Med 2015;162:W1-73.

24 Hingorani AD, Windt DAvander, Riley RD, et al. Prognosis research strategy (progress) 4: stratified medicine research. BMJ 2013;346:e5793.

25 Moons KGM, Wolff RF, Riley RD, et al. PROBAST: a tool to assess risk of bias and applicability of prediction model studies: explanation and elaboration. Ann Intern Med 2019;170:W1-33.

26 Boscainos PJ, Sapkas G, Stilianessi E, et al. Greek versions of the Oswestry and Roland-Morris disability questionnaires. Clin Orthop Relat Res 2003;411:40-53.

27 Billis E, Kapreli E, Krekoukias G, et al. Cross-Cultural validation of the sciatica bothersomeness index in a sample of Greek patients with sciatica. Physiotherapy 2015;101:e1448.

28 Georgoudes G, Papathanasiou G, Spyropoulos P. Physiotherapy assessment in painful musculoskeletal conditions: validity and reliability of the Greek Tampa scale of Kinesiophobia. Conference: International Forum on Pain Medicine. World Institute of Pain: European Federation of IASP Chapters, 2005. 
29 Lyrakos GN, Arvaniti C, Smyrnioti M, et al. Translation and validation study of the depression anxiety stress scale in the greek general population and in a psychiatric patient's sample. Eur. psychiatr. 2011;26:1731.

30 Batistaki C, Lyrakos G, Drachtidi K, et al. Translation, cultural adaptation, and validation of Leeds assessment of neuropathic symptoms and signs (LANSS) and Self-Complete Leeds assessment of neuropathic symptoms and signs (S-LANSS) questionnaires into the Greek language. Pain Pract 2016;16:552-64.

31 Bilika P, Neblett R, Georgoudis G, et al. Cross-Cultural adaptation and psychometric properties of the Greek version of the central sensitization inventory. Pain Pract 2020;20:188-96.

32 Georgoudis G, Watson PJ, Oldham JA. The development and validation of a Greek version of the short-form McGill pain questionnaire. Eur J Pain 2000;4:275-81.

33 Kontodimopoulos N, Pappa E, Niakas D, et al. Validity of SF-12 summary scores in a Greek general population. Health Qual Life Outcomes 2007;5:1-9.

34 Stynes S, Konstantinou K, Ogollah R, et al. Clinical diagnostic mode for sciatica developed in primary care patients with low back-related leg pain. PLoS One 2018;13:e0191852.
35 Foster NE. Physiotherapy added to GP care results in long-term improvements for sciatica. Aust J Physiother 2008;54:218.

36 Shmueli G. To explain or to predict? Statistical Science 2010;25:289-310.

37 Riley RD, Debray TPA, Collins GS, et al. Minimum sample size for external validation of a clinical prediction model with a binary outcome. Stat Med 2021;40:4230-51.

38 van Oort L, van den Berg T, Koes BW, et al. Preliminary state of development of prediction models for primary care physical therapy: a systematic review. J Clin Epidemiol 2012;65:1257-66.

39 Ridehalgh C, Sandy-Hindmarch OP, Schmid AB. Validity of clinical small-fiber sensory testing to detect Small-Nerve fiber degeneration. J Orthop Sports Phys Ther 2018;48:767-74.

40 Wellmon RH, Gulick DT, Paterson ML, et al. Validity and reliability of 2 Goniometric mobile Apps: device, application, and examiner factors. J Sport Rehabil 2015;25:371-9.

41 Sterne JAC, White IR, Carlin JB, et al. Multiple imputation for missing data in epidemiological and clinical research: potential and pitfalls. BMJ 2009;338:b2393.

42 Lewis RA, Williams NH, Sutton AJ, et al. Comparative clinical effectiveness of management strategies for sciatica: systematic review and network meta-analyses. Spine J 2015;15:1461-77. 